\title{
Un primer paso hacia el reconocimiento automático de escarabajos
}

\author{
Caleb Jiménez ${ }^{1}$, Maya Carrillo ${ }^{1}$, Hortensia Carrillo-Ruiz ${ }^{2}$, \\ María de Lourdes Sandoval S. ${ }^{1}$, Luis Alfredo Moctezuma ${ }^{1}$ \\ ${ }^{1}$ Benemérita Universidad Autónoma de Puebla, Facultad de ciencias de la computación, \\ Puebla, México \\ ${ }^{2}$ Benemérita Universidad Autónoma de Puebla, Facultad de Ciencias Biológicas, Puebla, \\ México \\ calebji@hotmail.com, cmaya@cs.buap.mx, \\ \{hortensia.carrillo, malosa1753, luisalfredomoctezuma\}@gmail.com
}

\begin{abstract}
Resumen. Los escarabajos pertenecen al orden Coleoptera, y son organismos importantes para el funcionamiento de los ecosistemas en los que habitan. Los taxónomos, clasifican a estos organismos para facilitar su identificación y estudio. Para ello emplean claves de identificación compuestas por dilemas, mismos que aumentan en número al acercarse a la categoría menos inclusiva. En este trabajo se presenta un método para identificar escarabajos de manera automática empleando como atributos, medidas estadísticas y la trasformada elíptica de Fourier. Se utilizaron 48 imágenes de 8 especies diferentes recolectadas en la región de Jolalpan en el estado de Puebla. Después de procesar las imágenes y extraer los atributos, se obtiene en la clasificación una exactitud del $89.58 \%$ utilizando Random Forest, con validación cruzada a 10 pliegues.
\end{abstract}

Palabras clave: identificación taxonómica, escarabajos, transformada elíptica de Fourier, medidas estadísticas.

\section{A First Step towards Automatic Beetle Recognition}

\begin{abstract}
Beetles belong to the order Coleoptera, and are important organisms for the ecosystems functioning in which they inhabit. Taxonomists classify these organisms to facilitate their identification and study. For this purpose, they use identification keys, composed by dilemmas, which increase in number when classification approaches to the least inclusive category. This work presents a method to automatically identify beetles using as attributes, statistical measures and the Elliptic Fourier Transform. 48 images of 8 different species collected in the Jolalpan region in Puebla state were used. After processing the images and extract the attributes, a classification accuracy of $89.58 \%$ was obtained using Random Forest, and 10-folds cross-validation.
\end{abstract}

Keywords: taxonomic identification, beetles, Fourier elliptic transform, statistical measures. 


\section{Introducción}

Dentro de la clase Insecta, el orden Coleoptera es uno de los grupos biológicos más diversos. Los escarabajos pertenecen a este orden y son organismos clave para el funcionamiento de los ecosistemas en los que habitan, ya que entre sus funciones se encuentra la degradación de la materia orgánica y su incorporación al suelo, favoreciendo así la circulación de la energía almacenada en los desechos orgánicos, esto los convierte en recicladores naturales. Además, estos insectos ocupan los primeros eslabones de la cadena trófica, siendo fuente importante de alimento para aves y mamíferos [1].

Debido a su morfología, colores llamativos e importancia ecológica, han sido estudiados, desde distintas áreas de la Biología, entre las que se encuentra la Sistemática. Los taxónomos elaboran esquemas de clasificación con el propósito de organizar a los diferentes organismos y de esta forma facilitar su estudio. Los sistemas de Clasificación consisten en categorías jerarquizadas, son una estructura de grupos inclusivos en donde cada grupo o categoría está definida por características compartidas entre los organismos, siendo en estos momentos la categoría más inclusiva reconocida en la Clasificación Biológica el Dominio y la menos inclusiva la Especie [2].

Con los sistemas de clasificación, los biólogos establecen relaciones de identidad entre un organismo particular y una categoría a la cual pertenece, es decir, un ejemplar puede identificarse taxonómicamente con un nombre (taxón) dentro de cada categoría. Para lograr esta relación de identidad, se deben construir claves de identificación para cada categoría a partir de una secuencia ordenada de dilemas o disyuntivas, donde cada dilema sucesivo va planteando dilemas cada vez más restringidos y finalmente se llega al nombre del grupo o taxón al que pertenece [2]. Por lo tanto, las claves de identificación resultan útiles al momento de determinar el nombre de alguna especie, sin embargo, la tarea de identificar no es fácil, ya que en las claves, conforme la tarea de clasificación se acerca a la categoría menos inclusiva (especie), los dilemas aumentan en número y complejidad, pudiendo ser más de 400 dilemas a resolver antes de poder determinar el nombre específico. Además, sólo pueden ser empleadas por especialistas, ya que se requiere de conocer los términos propios de la biología y de equipo especializado como microscopios estereoscópicos con un zoom mínimo de $0.5 x$.

Por lo anteriormente expuesto, se plantea este trabajo como un primer intento para generar una herramienta automática que facilite el trabajo de identificación de estos organismos, a partir de la transformada elíptica de Fourier para extraer características del borde del cuerpo del escarabajo y medidas estadísticas.

El presente artículo está organizado de la siguiente manera en la sección 2 se describen algunos trabajos relacionados, en la sección 3 se describe el método propuesto. En la sección 4 los experimentos y resultados y finalmente en la sección 5 las conclusiones y trabajo futuro.

\section{Trabajos relacionados}

Adams, et al. en [3] plantean un enfoque denominado Morfología geométrica. Este enfoque se basa en la digitalización de contornos o puntos clave, cuyas conformaciones 
espaciales son analizadas matemáticamente. Dentro de este grupo de técnicas, los métodos de contornos se basan en la digitalización de los puntos alrededor de un contorno para ajustarlos a una función matemática, generalmente derivada del análisis de Fourier. Posteriormente los coeficientes de éste, pueden emplearse en estudios comparativos.

Tabla 1. Ejemplares de escarabajos empleados en el estudio, se muestran los nombres que les corresponden dentro de cada una de las categorías taxonómicas a partir de familia.

\begin{tabular}{|c|c|c|c|c|}
\hline Familia & Subfamilia & Tribu & Género, subgénero y especie & $\begin{array}{c}\text { No. } \\
\text { ejemplares }\end{array}$ \\
\hline \multirow[t]{15}{*}{ Scarabaeidae } & Scarabaeinae & Scarabaeini & Canthon (Canthon) humectus & 6 \\
\hline & & & $\begin{array}{l}\text { Canthon (Canthon) } \\
\text { indigaceus }\end{array}$ & 6 \\
\hline & & & $\begin{array}{l}\text { Deltochilum gibbosum } \\
\text { sublaeve }\end{array}$ & 6 \\
\hline & & & Deltochilum tumidum & 5 \\
\hline & & Coprini & Copris incertus & 2 \\
\hline & & & Dichotomius colonicus & 6 \\
\hline & & & Dichotomius amplicollis & 1 \\
\hline & & & Ateuchus rodriguezi & 6 \\
\hline & & Phanaeini & Phanaeus mexicanus & 7 \\
\hline & & & $\begin{array}{c}\text { Coprophanaeus } \\
\text { (Coprophanaeus) pluto }\end{array}$ & 3 \\
\hline & & Onthophagini & Onthophagus lecontei & 1 \\
\hline & & & Onthophagus mextexus & 2 \\
\hline & & & Digitonthophagus gazella & 6 \\
\hline & Aphodiinae & Aphodiini & Labarrus pseudolividus & 2 \\
\hline & & Eupariini & Ataenius castaniellus & 3 \\
\hline Hybosoridae & Hybosorinae & & Hybosorus illigeri & 5 \\
\hline
\end{tabular}

Los descriptores elípticos de Fourier, propuestos por Kuhl y Giardina [4], pueden delinear cualquier forma con un contorno cerrado bidimensional. Estos han sido aplicados al análisis de varias formas biológicas.

Así, Rohlf y Archie en [5] presentan un análisis del contorno de las alas de 127 especies de mosquito del norte de México y comparan diferentes descriptores de Fourier, sin normalizar, normalizado, con coordenadas polares, entre otros y menciona que los descriptores de la trasformada elíptica de Fourier prometen resultados satisfactorios para la diferenciación entre especies.

Por otra parte, Furuta et al. en [6] analiza la forma de las hojas del frijol de soya empleando los coeficientes de los armónicos de la transformada elíptica de Fourier, emplea 20 armónicos normalizados y por lo tanto 77 descriptores por imagen de hoja. Con ellos realiza análisis de componentes principales y encuentra que la contribución acumulativa del quinto componente es del $96 \%$. Concluye que los coeficientes de 
Fourier proporcionan una medida cuantitativa poderosa para evaluar la forma de las hojas del frijol de soya.

Zhan y Wang en [7] utiliza la transformada elíptica de Fourier para analizar la forma del contorno del ala de cinco especies de Antlion (hormiga león parecida a la libélula). Ellos analizaron variaciones de la forma del perfil de alas de 5 especies del género: Considerando un total, 98 alas anteriores y 98 alas posteriores. Utiliza los veinte primeros armónicos de Fourier que resume a través de un análisis de componente principal y considera los primeros 8 componentes principales de la variación de forma para realizar pruebas estadísticas (análisis de varianza multivariable, análisis de variables canónicas y análisis de conglomerados). Concluye que sus resultados del análisis de Fourier de contorno de alas de las 5 especies están de acuerdo con el sistema taxonómico actual.

Recientemente los descriptores elípticos de Fourier, han sido empleados como para proponer la construcción de herramientas para la identificación automatizada de especies como lo mencionan Singh, et al. en [8] quienes han desarrollado un sistema automatizado de reconocimiento de especies de bambú basado en las características de forma de la vaina de Culm de bambú usando momentos de Fourier y de Legendre. Concluyen que el momento de Fourier tiene resultados significativamente mejores que el momento de Legendre, obteniendo un $100 \%$ de exactitud en la clasificación. El autor comenta que su sistema puede eliminar la necesidad de métodos laboriosos de reconocimiento humano que requieran un taxónomo de plantas. Los resultados obtenidos muestran una considerable precisión de reconocimiento, demostrando que las técnicas utilizadas son adecuadas para ser implementadas con fines comerciales.

Yang et al. en [9] presentan un trabajo para la identificación de insectos basado en el borde de sus alas reportando una exactitud media para la identificación de especies que varia entre el $90 \%$ y $98 \%$. Utilizan 120 ejemplares de 7 especies distintas. Emplean la transformada elíptica de Fourier con 30 armónicos y consideran 117 coeficientes para obtener las características del borde de las alas de estos insectos.

Para la clasificación emplean máquinas de soporte vectorial. Finalmente los descriptores elípticos de Fourier invariantes, simétricos, asimétricos y estandarizados, también se ha utilizados para clasificar granos en [10] Mebatsion et al., evalúan la forma de cuatro diferentes tipos de grano: cebada, avena centeno y trigo. Utilizan 100 imágenes por cada grano, los autores mencionan que los descriptores elípticos de Fourier ofrecen buenos resultados considerando la variabilidad de la forma de los granos y que podrían utilizarse para realizar clasificación no supervisada.

Hasta el momento no se tiene información de la utilización de los descriptores elípticos de Fourier para el estudio de las formas de los escarabajos.

\section{Metodología}

Los pasos para la identificación automática de escarabajos son: (1) adquisición de imágenes, (2) preprocesamiento de imágenes, (3) extracción de características y (4) clasificación de los datos. Pasos que se describen en las secciones subsecuentes. 


\subsection{Adquisición de imágenes}

Las imágenes fueron obtenidas de un grupo de ejemplares de colección provenientes de la región de Jolalpan en el estado de Puebla, estos ejemplares fueron recolectados durante un año en esta zona, su captura no es sencilla por lo que son pocos los representantes empleados en este estudio [11]. En total se emplearon 67 especímenes conservados en seco, los cuales fueron previamente identificados de manera tradicional empleando las claves de identificación para las especies de escarabajos del estado de Puebla [12] y pertenecen a dos familias, tres subfamilias, seis tribus, 12 géneros y 16 especies (Tabla 1).

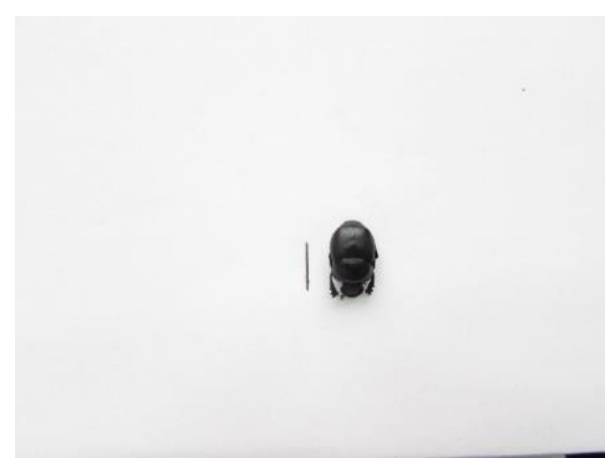

Fig. 1. Escarabajo en posición dorsal y escala de referencia de diez milímetros.

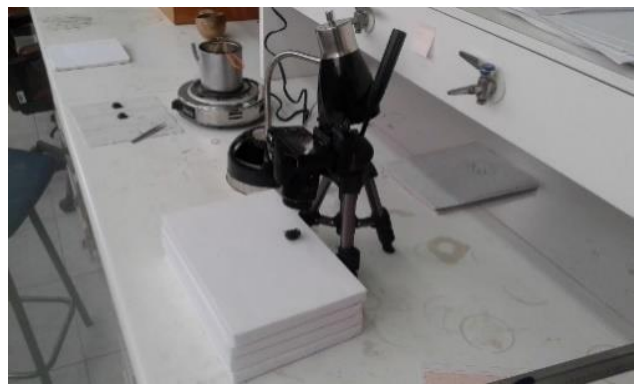

Fig. 2. Obtención de imágenes de los ejemplares estudiados.

Para obtener las imágenes, cada uno de los ejemplares se reblandecieron con vapor de agua para poder retirar el alfiler entomológico que se emplea para conservarlos. Posteriormente fueron colocados en posición dorsal sobre una placa de unicel forrada con papel blanco, sobre la cual se dibujó una escala de referencia en milímetros (Fig. 1). Finalmente fueron fotografiados con una cámara canon 1068C001AA montada sobre un tripie (Fig. 2).

\subsection{Pre-procesamiento de imágenes}

Para extraer las características de las imágenes, estas deben pre-procesarse previamente, para ello: Se recortaron los escarabajos rodeados por un margen de 15 
pixeles, figura 3a; se convirtieron a escala de grises y se bina-rizaron figura $3 b$. Posteriormente, se eliminaron las regiones menores de 100 pixeles, figura 3c. Estos 100 pixeles se determinaron en función de la línea de referencia, figura 1, misma que tiene 104 pixeles. Mantener dicha línea será de utilidad en futuros experimentos.

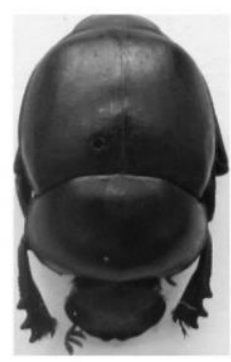

a)

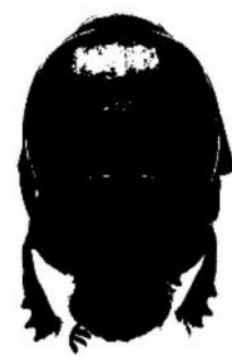

b)

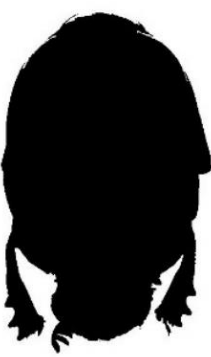

c)

Fig. 3. Pre-procesamiento de las imágenes de un ejemplar de la especie Canthon (Canthon) humectus: a) imagen recortada, b) imagen bina-rizada, c) imagen después de eliminar regiones menores de 100 pixeles.

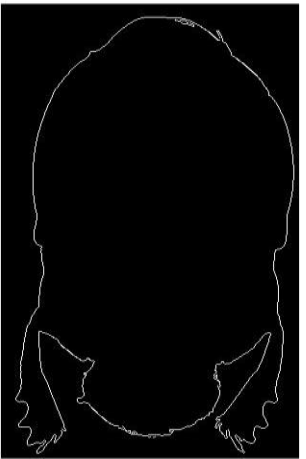

a)

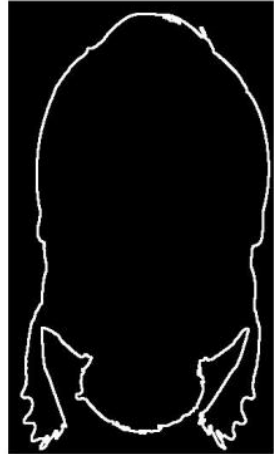

b)

Fig. 4. Imagen de un ejemplar de la especie Canthon (Canthon) humectus a) aplicación de mascara sobel b) aplicación de la operación de dilatación.

Posteriormente se procedió a detectar el borde de las imágenes aplicando el algoritmo Sobel de detección de borde, definido de manera muy simple debido a que solo utilizamos ocho puntos de la imagen alrededor del punto analizar ya que buscamos un cambio abrupto en nuestra imagen bina-rizada que representa el borde de nuestra imagen figura 4.

Finalmente se aplicó la operación morfológica de dilatación que consiste en adicionar pixeles a la frontera de la imagen, el procedimiento consiste en analizar la imagen por punto considerando sus ocho vecinos más cercanos y tener un contorno completamente delimitado, utilizamos la función de Matlab imdilate, figura $4 \mathrm{~b}$.

Las coordenadas $(\mathrm{x}, \mathrm{y})$, puntos del contorno, se emplearon para obtener las características para el proceso de clasificación. 


\subsection{Extracción de características}

Las coordenadas $(\mathrm{x}, \mathrm{y})$ obtenidas en el paso anterior se utilizaron para calcular descriptores elípticos de Fourier. Dichos descriptores fueron calculados empleando códigos de cadena para describir los contornos de las imágenes como se describe en [4]. El algoritmo no requiere que lo puntos sean equidistantes, y puede describir cualquier contorno cerrado siempre y cuando el número de armoniacos sea el adecuado. Los coeficientes, para el n-ésimo armónico de la expansión elíptica de Fourier de la secuencia de coordenadas $\mathrm{x}$, son:

$$
\begin{aligned}
& \mathrm{A}_{\mathrm{n}}=\frac{\mathrm{T}}{2 \mathrm{n}^{2} \pi^{2}} \sum_{\mathrm{p}=2}^{\mathrm{k}} \frac{\Delta \mathrm{x}_{\mathrm{p}}}{\Delta \mathrm{t}_{\mathrm{p}}}\left(\cos \left[2 \mathrm{n} \pi \mathrm{t}_{\mathrm{p}} / \mathrm{T}\right]-\cos \left[2 \mathrm{n} \pi \mathrm{t}_{\mathrm{p}-1} / \mathrm{T}\right]\right. \\
& \mathrm{B}_{\mathrm{n}}=\frac{\mathrm{T}}{2 \mathrm{n}^{2} \pi^{2}} \sum_{\mathrm{p}=2}^{\mathrm{k}} \frac{\Delta \mathrm{x}_{\mathrm{p}}}{\Delta \mathrm{t}_{\mathrm{p}}}\left(\sin \left[2 \mathrm{n} \pi \mathrm{t}_{\mathrm{p}} / \mathrm{T}\right]-\sin \left[2 \mathrm{n} \pi \mathrm{t}_{\mathrm{p}-1} / \mathrm{T}\right]\right.
\end{aligned}
$$

donde $\mathrm{k}$ es el número de pasos (segmentos) en el trazo del contorno, $\Delta \mathrm{x}_{\mathrm{p}}$ es el desplazamiento en el eje $\mathrm{x}$ entre los pasos $\mathrm{p}-1$ y $\mathrm{p}, \Delta \mathrm{t}_{\mathrm{p}}$ es la longitud del segmento entre los pasos p-1 y p, $t_{\mathrm{p}}$ es la longitud acumulada de los segmentos y $\mathrm{T}=\mathrm{t}_{\mathrm{k}}$ es la longitud total del contorno aproximado por el trazo de un polígono. Los coeficientes para la coordenada y, $C_{n}$ y $D_{n}$ se calculan de igual manera empleando los datos para el eje y.

Los coeficientes calculados se normalizaron para hacerlos invariantes al tamaño, a la rotación, y punto de inicio, como se describe en [4]. Después de la normalización los primeros tres coeficientes del primer armónico son constantes y por lo tanto no considerados como atributos. El resto de coeficiente se utilizaron como atributos en el proceso de clasificación.

Por otra parte también se calcularon medidas estadísticas, considerando las coordenadas x e y, estas fueron: a) media, b) mediana, c) máximo, d) mínimo, e) suma, f) desviación estándar, g) varianza, h) curtosis, i) oblicuidad.

\subsection{Clasificación de datos}

Después de extraer los atributos para cada imagen, estos fueron los datos de entrada para tres clasificadores: a) Máquinas de soporte vectorial (Support vector machine, SVM) que es de aprendizaje supervisado, Bayes ingenuo (Naive Byes) donde el aprendizaje es supervisado y Selvas aleatorias (Random Forest) que es de aprendizaje no supervisado.

Estos clasificadores fueron ejecutados primero considerando sólo los atributos estadísticos, es decir 18 atributos por imagen. Después los coeficientes de los armónicos de la transformada elíptica de Fourier y finalmente ambos conjuntos. Se utilizó validación cruzada a 10 pliegues. En la siguiente sección se muestran los resultados obtenidos. 


\section{Experimentos y resultados}

Para realizar los experimentos se consideraron únicamente las especies de la familia Scarabaeidae, que tienen 5 ejemplares o más para un total de 48 imágenes, para este trabajo la orientación de la imagen es importante pues en la extracción de coordenadas para las medidas estadísticas se consideran las imágenes en una misma posición que es vertical con la masa antenal en la parte inferior izquierda. Para estas imágenes se calcularon las medidas estadísticas mencionadas en la sección 3.3. Los resultados se muestran en la Tabla 2.

Tabla 2. Resultados de la exactitud obtenidos para las imágenes de la familia Scarabaeidae caracterizadas con medidas estadísticas.

\begin{tabular}{cccc}
\hline No. puntos & Random Forest & SVM & Naive Bayes \\
\hline 30 & $\mathbf{8 3 . 3 4}$ & 77.08 & $\mathbf{8 3 . 3 4}$ \\
50 & 83.34 & 83.34 & 75.00 \\
100 & 83.34 & 83.34 & 85.42 \\
250 & 81.25 & 79.17 & 79.17 \\
500 & 79.17 & 83.34 & 83.34 \\
\hline
\end{tabular}

Como puede observarse en la Tabla 2, ocho resultados alcanzan una exactitud del $83.33 \%$, dos de ellos empleando 18 atributos.

Tabla 3. Resultados de la exactitud obtenidos para las imágenes de la familia Scarabaeidae caracterizadas con los coeficientes de los armónicos de la transformada elíptica de Fourier.

\begin{tabular}{ccccc}
\hline No.armonico & No.puntos & Random Forest & SVM & Naive Bayes \\
\hline 2 & 30 & 47.92 & 12.50 & 47.92 \\
2 & 50 & 47.92 & 12.50 & 47.92 \\
2 & 100 & 47.92 & 12.50 & 47.92 \\
4 & 30 & 41.67 & 22.92 & $\mathbf{5 2 . 0 8}$ \\
4 & 50 & 41.67 & 22.92 & 52.08 \\
4 & 100 & 41.67 & 22.92 & 52.08 \\
8 & 30 & 35.42 & 39.58 & 41.67 \\
8 & 50 & 35.42 & 39.58 & 41.67 \\
8 & 100 & 35.42 & 39.58 & 41.67 \\
16 & 30 & 18.75 & 29.17 & 29.17 \\
16 & 50 & 18.75 & 29.17 & 29.17 \\
16 & 100 & 18.75 & 29.17 & 29.17 \\
\hline
\end{tabular}


Posteriormente se clasificaron los mismos ejemplares pero ahora calculando los coeficientes para los armónicos de la transformada elíptica de Fourier, variando el número de puntos y armónicos, los resultados obtenidos se muestran en la Tabla 3.

En la Tabla 3, la exactitud de la clasificación no se altera variando el número de puntos. Pero si al variar el número de armónicos. La mejor exactitud se obtiene con 4 armónicos, lo que nos genera 13 atributos, con el clasificador Naive Bayes y 30 puntos, lo implican menos tiempo de procesamiento según nuestra tabla.

Nuestras tablas muestran que estadísticamente tenesmos mejores resultados comparados con los descriptores de Fourier y esto se debe a que la forma de nuestros escarabajos no es totalmente elíptica pues en la parte de la cabeza cuenta con la maza antenal, la tibia y el tarsus.

Finalmente se combinaron los atributos estadísticos con los descriptores de Fourier, considerando los resultados con 30 puntos pues aportan la misma exactitud y menor tiempo de procesamiento. Los resultados están en la Tabla 4.

Tabla 4. Resultados de la exactitud obtenidos para las imágenes de la familia Scarabaeidae combinando atributos estadísticos y descriptores de Fourier.

\begin{tabular}{cccccc}
\hline $\begin{array}{c}\text { No. puntos } \\
\text { Estadistica }\end{array}$ & $\begin{array}{c}\text { No. } \\
\text { armónicos }\end{array}$ & $\begin{array}{c}\text { No.puntos } \\
\text { descriptor }\end{array}$ & Random Forest & SVM & $\begin{array}{c}\text { Naive } \\
\text { Bayes }\end{array}$ \\
\hline 30 & 2 & 30 & 85.42 & 81.25 & 79.17 \\
30 & 4 & 30 & 87.50 & 83.34 & 87.50 \\
30 & 8 & 30 & $\mathbf{8 9 . 5 8}$ & 81.25 & 83.34 \\
30 & 16 & 30 & 81.25 & 75.00 & 79.17 \\
\hline
\end{tabular}

Como puede observarse en la Tabla 4, la exactitud de la clasificación mejora cuando se combinan los atributos, obteniendo una mayor exactitud igual a $89.58 \%$ con Random Forest, 30 puntos para calcular las medidas estadísticas y los descriptores de Fourier. En la Tabla 5 se muestra la matriz de confusión para estos resultados donde puede observarse que los errores se dan entre las especies Deltochilum gibbosum sublaeve y Deltochilum tumidum que de acuerdo a la Tabla 1 pertenecen a la misma tribu. En la Tabla 6 se presentan los resultados para la precisión, recuerdo y medida F donde puede observarse que el promedio de la precisión es de 0.90 y de medida F de 0.89 .

Tabla 5. Matriz de confusión para la clasificación obtenida.

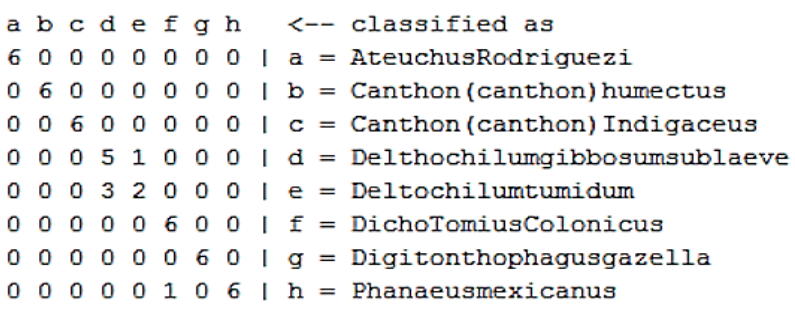


Tabla 6. Precisión, recuerdo y medida F obtenidos.

\begin{tabular}{lccc}
\hline \multicolumn{1}{c}{ Especie } & Precisión & Recuerdo & Medida F \\
\hline Ateuchus rodriguezi & 1.00 & 1.00 & 1.00 \\
Canthon (Canthon) humectus & 1.00 & 1.00 & 1.00 \\
Canthon (Canthon) indigaceus & 1.00 & 1.00 & 1.00 \\
Deltochilum gibbosum sublaeve & 0.63 & 0.83 & 0.71 \\
Deltochilum tumidum & 0.67 & 0.40 & 0.50 \\
Dichotomius colonicus & 0.86 & 1.00 & 0.92 \\
Digitonthophagus gazella & 1.00 & 1.00 & 1.00 \\
Phanaeus mexicanus & 1.00 & 0.86 & 0.92 \\
Phanaeus mexicanus & 1.00 & 0.86 & 0.92 \\
\hline Promedio & 0.90 & 0.89 & 0.89 \\
\hline
\end{tabular}

En la Figura 5 se muestra la aproximación al contorno de un ejemplar con 30 puntos y 8 armónicos es decir 29 atributos, puede observarse que aún hay imprecisiones considerables.

\section{Conclusiones y trabajo futuro}

Las medidas estadísticas proporcionan mejor exactitud al clasificar los escarabajos empleando 18 atributos contra 29 empleados con la transformada elíptica de Fourier con 8 armónicos. Si bien los descriptores de Fourier alcanzan un máximo de $52 \%$ de exactitud pensamos que esto se debe al escaso número de imágenes con que contamos, únicamente 48, mientras que en el trabajo relacionado se cuenta con más de 100 imágenes

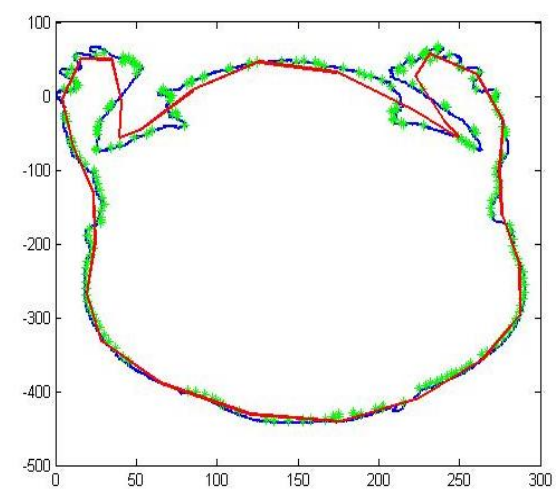

Fig. 5. Aproximación al contorno de una ejemplar de la especie Canthon (Canthon) humectus empleando la transformada elíptica de Fourier. 
Los errores en la clasificación se dan entre ejemplares de la misma tribu, lo que implica que de manera natural tienen ciertos atributos en común, así que estos errores deben disminuir al contar con más ejemplares. En cinco especies la precisión es igual a 1 , con el 100 por ciento de ejemplares clasificados correctamente. Como trabajo futuro se experimentará agregando nuevos ejemplares y otros atributos como el color e identificación de líneas o manchas específicas de los ejemplares.

\section{Bibliografía}

1. Castañeda-Osorio, R., Carrillo-Ruiz, H., Rivas-Arancibia, S. P., Sánchez- Carrillo, M.: Melolonthidae y Cetoniidae (Coleoptera: Scarabaeoidea) en el Rancho El Salado, Jolalpan, Puebla, México. Dugesiana, 22(2), pp. 227-241 (2015)

2. Morrone, J. J.: Sistemática. Fundamentos, métodos, aplicaciones. Facultad de Ciencias, UNAM, Ciudad de México (2013)

3. Adams, D. C., Rohlf, F. J., Slice, D. E.: Geometric morphometrics: ten years of progress following the 'revolution'. Italian Journal of Zoology, 71, pp. 5-16 (2004)

4. Kuhl, F. P., Giardina, C. R.: Elliptic Fourier features of a closed contour. Computer graphics and image processing, 18, pp. 236-258 (1982)

5. Rohlf, F. J., Archie, J. W.: A comparison of Fourier methods for the description of wing shape in mosquitoes (Diptera: Culicidae). Systematic Zoology, 33, pp. 302-317 (1984)

6. Furuta, N., Ninomiya, S., Takahashi, S., Ohmori, H., Ukai, Y.: Quantitative evaluation of soybean (Glycine max L. Merr.) leaflet shape by principal component scores based on elliptic Fourier descriptor. Breeding Science, 45, pp. 315-320 (1995)

7. Zhan, Q. B., Wang, X. L.: Elliptic Fourier Analysis of the Wing Outline Shape of Five Species of Ant- lion (Neuroptera: Myrmeleontidae: Myrmeleontini). Zoological Studies, 51(3), pp. 399-405 (2012)

8. Singh, K., Gupta, I., Gupta, S.: Classification of Bamboo Species by Fourier and Legendre Moment. International Journal of Advanced Science and Technology, 50, pp. 61-70 (2013)

9. Yang, H. P., Ma, C. S., Wen, H., Zhan, Q. B., Wang, X. L.: A tool for developing an automatic insect identification system based on wing outlines. Scientific reports (2015)

10. Mebatsion, H. K., Paliwal, J., Jayas, D. S.: Evaluation of variations in the shape of grain types using principal components analysis of the elliptic Fourier descriptors. Computers and electronics in agriculture, 80 (2012)

11. Sánchez-Velázquez, B., Carrillo-Ruiz, H., Morón, M. Á., Rivas-Arancibia, S. P.: Especies de Scarabaeidae e Hybosoridae (Coleoptera: Scarabaeoidea) que habitan en la comunidad del Rancho El Salado, Jolalpan, Puebla, México. Dugesiana, 18(2), pp. 207-215

12. Morón, M. A., Aragón-García, A., Carrillo-Ruiz, H.: Fauna de escarabajos del estado de Puebla. Publicado por MA Morón, Coatepec, Veracruz, México (2013) 